\title{
Influence of bonded abrasive particles size on wear of polymeric particle composites based on waste
}

\section{Petr Valášek, Miroslav Müller}

Department of Material Science and Manufacturing Technology, Faculty of Engineering, Czech University of Life Sciences in Prague. E-mail: valasekp@tf.czu.cz.

Experiments described in this paper determine two body abrasion of two- and three-phase polymeric particle composites with a different size of filler particles. An epoxy resin was the matrix of composite systems, corundum and chips of iron metals represented the filler created from secondary raw materials. The results show clear influencing of composite hardness and abrasive wear resistance caused by the fillers. The influence of the size of bonded abrasive particles on the wear of the composites based on the waste was proved and it is noticeable with tested abrasive clothes it is 65 times.

Keywords: composite system, secondary raw materials, wear

\section{Acknowledgement \\ This paper has been done when solving the IGA TF grant. (nr. 31140/1312/3104) \\ References}

[1] BERTHELOT, Jean M. Composite Materials - Mechanical Behavior and Structural Analysis. Mechanical engineering series: Springer New York, 1999. 635 p. ISBN 0-387-98426-7.

[2] SUCHÁNEK J.; KUKLÍK, V.; ZDRAVECKÁ, E. Abrazivní opotřebení materiáli̊, Prague, 2007. 162 p. ISBN 978-80-01-03659-4

[3] VALÁŠEK Petr; MÜLLER Miroslav.; KEJVAL Jiří: Faktory limitující použití polymerních částicových kompozitů - pórovitost. Strojírenská technologe, 2012, roč. XVII, č. 3, p. 192 - 197. ISSN 1211- 4162.

[4] MÜLLER Miroslav; VALÁŠEK Petr et al. Aplikace návarů a kompozitů v oblasti technologie pěstování a sklizně cukrové řepy. Listy cukrovarnické a řepařské, 2011, vol. 9, p. 304 - 307. ISSN 1210-3306.

[5] SATAPATHY B.K.; BIJWE, J. Analysis of simultaneous influence of operating variables on abrasive wear of phenolic composites. Wear, 2002, vol. 253, p. 787-794. ISSN 0043-1648.

[6] MOHAN N.; NATARAJAN S.; KUMARESHBABU S. P. The role of synthetic and natural fillers on threebody abrasive wear behaviour of glass fabric-epoxy hybrid composites. Journal of applied polymer science, 2012, 124, p. 484-494. ISSN 1097-4628.

[7] BASAVARAJAPPA S.; JOSHI A. G.; ARUN K.; KUMAR A.P.; KUMAR M.P. Three-Body Abrasive Wear Behaviour of Polymer Matrix Composites Filled with SiC Particles. Polymer-Plastics Technology and Engineering, 2010, 14, p. 8-12. ISSN 0360-2559.

[8] NOVÁK Martin. Surface quality hardened steels after grinding. Manufacturing technology. 2011, 11, p. 55-59. ISSN 1213-2489.

[9] MÜLLER Miroslav; VALÁŠEK Petr. Abrasive wear effect on Polyethylene, Polyamide 6 and polymeric particle composites. Manufacturing Technology, 2011, 11, p. 55-59. ISSN1213-2489.

[10] ČSN EN ISO 2039-1. Plastics - Determination of hardness - Part 1: Ball indentation Metod, 2000.

[11] ISO 62 1466. Rubber; Determination of abrasion resistance using a rotating cylindrical drum device, 1985.

[12] XIA JIA; XIA OMEI LING. Influence of $\mathrm{Al}_{2} \mathrm{O}_{3}$ reinforcement on the abrasive wear characteristic of $\mathrm{Al}_{2} \mathrm{O}_{3} / \mathrm{PA} 1010$ composite coatings. Wear, 2005, 258, p. 1342-1347. ISSN 0043-1648.

[13] STEWART, I.; CHAMBERS A.; GORDON T. The cohesive mechanical properties of a toughened epoxy adhesive as a function of cure level. International Journal of Adhesion and Adhesives, 2006, 24, p. 277-287. ISSN 0143-7496. 